Journal of Case Reports 2018;8(2):150-153

\title{
Sirolimus Therapy for Renal Masses in Tuberous Sclerosis Complex
}

\author{
Shashanka Dhanuka, Aruni Ghose, Sohini Samaddar \\ Department of Urology, Nil Ratan Sircar Medical College and Hospital, Kolkata, West Bengal, India.
}

Corresponding Author:

Dr. Shashanka Dhanuka

Email: drdhanuka@gmail.com

This is an Open Access article distributed under the terms of the Creative Commons Attribution License (creativecommons.org/ licenses/by/3.0).

Received : January 22, 2018

Accepted : May 15, 2018

Published : June 15, 2018

\begin{abstract}
Background: We report a rare case of tuberous sclerosis complex with bilateral renal masses that was managed conservatively using mTOR inhibitors. Case Report: A 18 year old girl presented with right flank pain and features of tuberous sclerosis complex. Imaging revealed bilateral, large, heterogenous, enhancing renal masses. The patient was successfully managed with sirolimus and is on regular follow up with no complications. Conclusion: We conclude that renal masses in tuberous sclerosis complex can be managed on sirolimus.
\end{abstract}

Keywords: Angiomyolipoma, Flank Pain, Hematuria, Kidney Neoplasms, Sirolimus, Tuberous Sclerosis.

\section{Introduction}

Tuberous sclerosis complex (TSC) is a rare autosomal dominant genetic disease (with an incidence of 1 in 5,000 to 10,000 live births) with incomplete penetrance. It is a neurocutaneous syndrome classically characterised by a triad of mental retardation, epilepsy and various skinlesions. The renal affections include angiomyolipomas (about $80 \%$ ), cysts (about 15\%) or renal cell carcinomas (about 4\%) and are considered to be the leading cause of death in patients with TSC [1]. Angiomyolipomas greater than $4 \mathrm{~cm}$ require angioembolization as it may cause hemorrhage and present with hematuria [2]. Here we present a case of tuberous sclerosis with multiple, large, bilateral renal masses without any hematuria or complications limiting her quality of life.

\section{Case Report}

An 18 year old girl presented with right flank pain and a lump in the right side of the abdomen for one and a half months. The pain was insidious in onset, dull aching in character, non-radiating, exacerbated by straining, relieved by rest, non-progressive and mild to moderate in severity, not associated with any other symptoms. She noticed a diffuse lump in the right side of the abdomen which did not change in size over this period. There was no history of hematuria, lower urinary tract symptoms, fever with chills or rigor, anorexia, weight loss or drug allergies. Seizures, loss of consciousness and vision abnormalities were absent. Her social and communication skills were suboptimal whereas her intellectual development was optimum. She is nondiabetic and non-hypertensive. Her father is also a known case of tuberous sclerosis. No other member of the family is suffering from similar complaints. On general examination she was of average built and nutrition. She had mild pallor. There was no icterus, cyanosis, clubbing, lymphadenopathy, edema or engorged neck veins. Her vital signs were within normal limits. An adenoma sebaceum on the malar region of the face bilaterally and a Shagreen patch over the lower back were found. On per abdominal examination fullness on the right side of the abdomen was detected on inspection. A diffuse, tender, non-reducible, non-compressible mass measuring about $12 \mathrm{~cm}$ in the vertical axis was palpable in the right hypochondrial, lumbar 


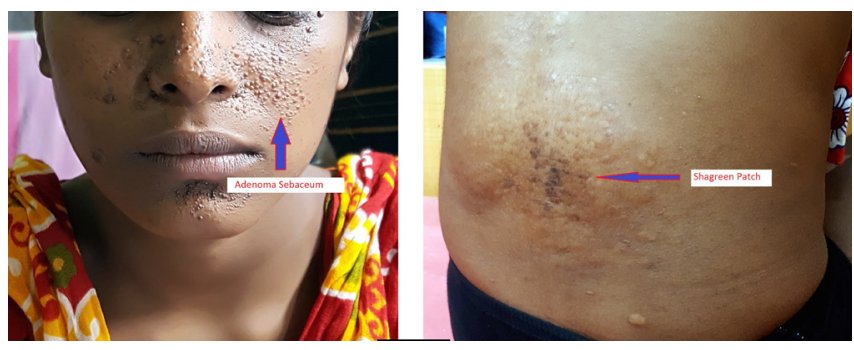

Fig.1: Clinical image of adenoma sebaceum and Shagreen patches.

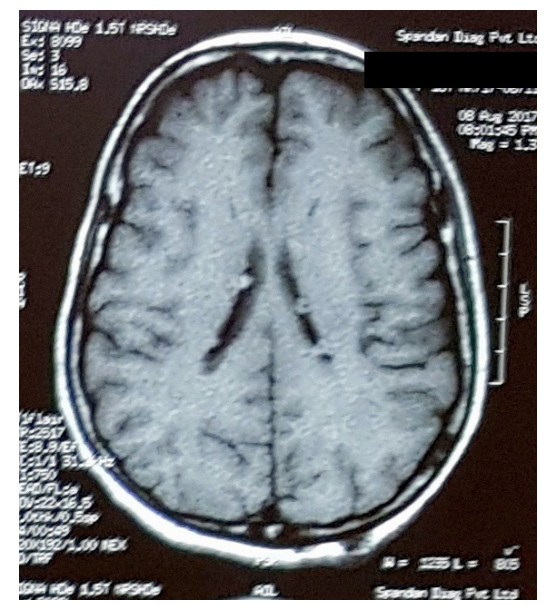

Fig.2: MRI brain showing subcortical tubers.

and iliac regions. It was ballotable, firm with margins ill-defined, smooth surface, not fixed to the adjacent structures and moving with respiration. No other palpable abdominal lumps, organomegaly or shifting dullness were elicited. The mass was dull on percussion and a band of colonic resonance was appreciated. Normal bowel sounds were heard on auscultation and venous hum or bruit was absent. A fundoscopy revealed a pigmentary naevus in the infero-nasal peripheral retina of the left eye. Dental examination revealed enamel pitting. Other systems were within normal limits. A complete blood count revealed hemoglobin of $8.6 \mathrm{gm} / \mathrm{dL}$ and $\mathrm{RBC}$ count of $2.9 \mathrm{million} / \mathrm{cc}$, while the other parameters were normal. Serum urea and creatinine were $22 \mathrm{mg} / \mathrm{dL}$ and $0.75 \mathrm{mg} / \mathrm{dL}$ respectively. Serum electrolytes and blood sugar were within normal limits. Urine routine examination was normal and urine culture showed no growth of microorganism. ECG showed
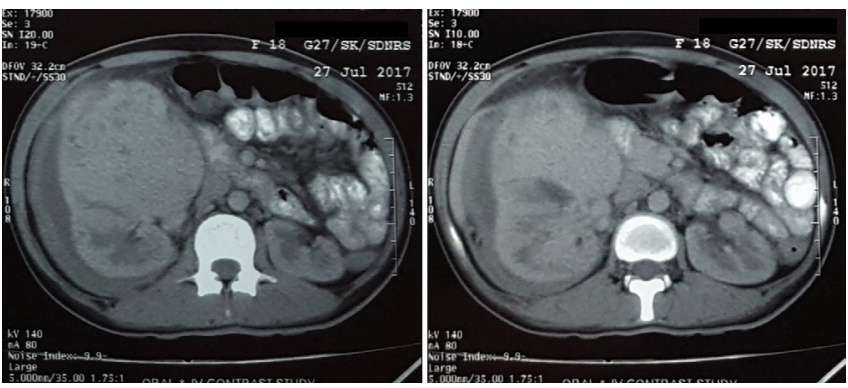

Fig.3: CT scan KUB showing bilateral renal masses.

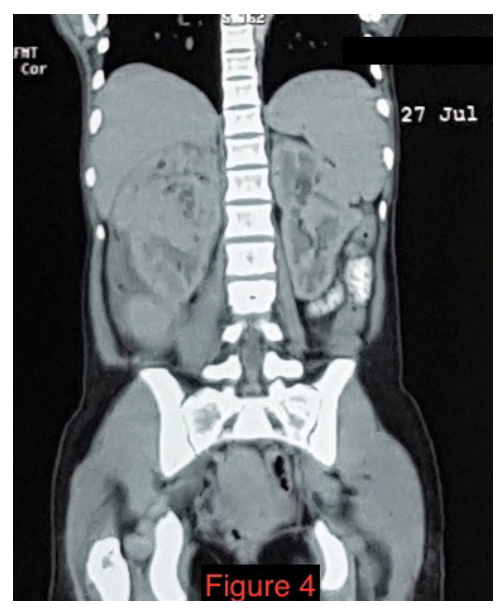

Fig.4: CT scan KUB showing bilateral renal masses (coronal reconstrunction).

sinus rhythm. No abnormality was detected on chest $\mathrm{X}$ ray. An ultrasonography of the abdomen showed the presence of heterogeneous hyper-echoic mass with cystic and solid components in the right kidney and a mild, bulky left kidney. A contrast enhanced CT scan of whole abdomen suggested large nodular heterogeneously enhancing lesions in the right kidney (largest measuring $9.4 \times 12.9 \times$ $10.0 \mathrm{~cm}$ ), showing focal areas of fatty attenuation. Similar lesions were also noted in the left kidney (largest measuring $2.7 \times 2.9 \times 3.7 \mathrm{~cm}$ ). It also showed multiple sclerotic lesions in the bone. An MRI scan of brain (plain and contrast) showed multiple tiny cortical and subcortical tubers in frontal, temporal and parietal lobes bilaterally; multiple subependymal micro-calcifications and giant cell astrocytomas in lateral ventricles. Patient was admitted to the urology ward and investigated. She was counselled about the option of prophylactic 
angio-embolization or nephrectomy for which she did not consent. She was then offered an alternative mode of treatment in the form of tablet sirolimus $2 \mathrm{mg}$ per day. She was also prescribed iron folic acid tablets. There were no adverse effects to this treatment. Patient was followed up after 3 months in the outpatient department. There was no history of pain, new onset hematuria or change in the size or nature of the lump. On examination, there was no tenderness over the lump and other findings were similar to those before the initiation of treatment. The ultrasonography of whole abdomen did not reveal any retroperitoneal hemorrhage, fresh peri-renal collection, or change in characteristics of the lesion. Serum urea and creatinine were $17 \mathrm{mg} / \mathrm{dL}$ and $1.0 \mathrm{mg} / \mathrm{dL}$ respectively. Hemoglobin was $9.7 \mathrm{gm} / \mathrm{dL}$ and RBC count was 3.6 million/cc. Subsequently she was followed up at 6 months and 1 year, post-initiation of treatment and she is doing well.

\section{Discussion}

Renal manifestations occur with a high frequency and a wide range of severity in TSC. Approximately $80 \%$ of patients with TSC develop renal angiomyolipomas, $15 \%$ of them present with renal cysts and 4\% develop renal cell carcinomas. Angiomyolipoma (AML) is a mesenchymal tumor of the kidney, composed of vascular, smooth muscle and fat elements. Since patients are usually asymptomatic, the diagnosis of AML is often incidental; this happens with lesions with a diameter of less than $4 \mathrm{~cm}$. Lesions greater than 4 $\mathrm{cm}$ in diameter are often symptomatic and manifest with a clinical picture characterized by lumbar pain, anaemia and hematuria. Retroperitoneal hemorrhage and/or bleeding into the renal collecting system are the major complications of AML; both conditions may put the patient's life at risk [3]. The therapeutic strategy varies from case to case: selective embolization of the renal artery and surgical removal of the lesion are the pillars of
AML management. Nephrectomy can be opted for in more severe cases. In case the aforementioned alternatives cannot be performed, a medical approach with hormonal therapy or with agents such as sirolimus, an inhibitor of the mammalian target of rapamycin (mTOR), can be chosen. It has shown to decrease the size, vascularity and symptoms associated with such lesions [4].

Although in our case the patient had a large renal mass, which could be AML, the surprising lack of hematuria or other complications as well as the unwillingness of the patient to undergo a procedure, prompted us to keep her under close surveillance. We offered prophylactic angioembolization to our patient and explained the possible complications of this procedure including bleeding and renal infarction. Given her young age, the presence of bilateral renal masses and the possible complications of this procedure, she refused to undergo angio-embolization and chose to be managed conservatively with medication and follow up. The patient is on sirolimus and we are following her up with ultrasound every three months. At one year follow up, she is doing well and has no episode of hematuria or other associated complications.

\section{Conclusion}

Despite guidelines suggesting prophylactic surgery of large renal masses in TSC, we have seen that such patients can be managed safely with mTOR inhibitor along with close follow up, with appropriate imaging. They need not undergo an intervention unless there is an absolute indication. We also conclude that renal masses associated with TSC tend to be benign.

Contributors: SD: manuscript writing, patient management; AG, SS: manuscript editing, patient management. SD will act as guarantor. All authors approved the final version of this manuscript.

Funding: None; Competing interests: None stated. 


\section{References}

1. Rakowski SK, Winterkorn EB, Paul E, Steele DJ, Halpern EF, Thiele EA. Renal manifestations of tuberous sclerosis complex: Incidence, prognosis, and predictive facrors. Kidney Int. 2006;70(10):1777-1782.

2. Kothary N, Soulen MC, Clark TW, Wein AJ, ShlanskyGoldberg RD, Crino PB, Stavropoulos SW. Renal AML: Long-term results after angioembolization. J Vasc Interv Radiol. 2005;16:45-50.
3. Urciuoli P, D’Orazi V, Livadoti G, Foresi E, Panunzi A, Cialini M. Treatment of renal angiomyolipoma: surgery versus angioembolization. G Chir. 2013;34:326-331.

4. Bissler JJ, McCormack FX, Young LR, Elwing JM, Chuck G, Leonard JM, et al. Sirolimus for angiomyolipoma in tuberous sclerosis complex or lymphangioleiomyomatosis. $\mathrm{N}$ Engl $\mathrm{J}$ Med. 2008;358:140-151. 